\title{
Agôn
}

Revue des arts de la scène

$8 \mid 2019$

Matières

\section{Face à la matière : parcours sensible et interrogations éparses d'une marionnettiste}

\section{Dinaïg Stall}

\section{(2) OpenEdition \\ 1 Journals}

Édition électronique

URL : http://journals.openedition.org/agon/5892

DOI : $10.4000 /$ agon.5892

ISSN : 1961-8581

Éditeur

Association Agôn

Référence électronique

Dinaïg Stall, «Face à la matière : parcours sensible et interrogations éparses d'une marionnettiste », Agôn [En ligne], 8| 2019, mis en ligne le 01 décembre 2019, consulté le 04 juin 2020. URL : http:// journals.openedition.org/agon/5892 ; DOI : https://doi.org/10.4000/agon.5892

Ce document a été généré automatiquement le 4 juin 2020.

Association Agôn et les auteurs des articles 


\title{
Face à la matière : parcours sensible et interrogations éparses d'une marionnettiste
}

\author{
Dinaïg Stall
}

\section{1. Être matière}

1 En tant que praticienne des arts de la marionnette, je ne peux que m'interroger sur la place de la matière dans ma démarche artistique et pédagogique : mon travail consiste à la modeler, l'animer, la guider - et à transmettre à d'autres des façons possibles de le faire. La toucher et me laisser toucher. Être à l'écoute de ses propositions (peut-on appeler cela ainsi ? Suis-je déjà en train de projeter une intentionnalité humaine ?). Je suis de plus en plus sensible à cette approche qui consiste à sortir du brouhaha des injonctions, souvent contradictoires, quant à ce qu'il faudrait faire avec la matière pour qu'elle puisse être qualifiée de "marionnettique », quant aux formes à lui donner, afin de chercher plutôt à découvrir celles qu'elle porte en elle.

2 Comme l'écrit Eric Bass, directeur du Sandglass Theatre ${ }^{1}$, « En tant que marionnettistes, ce qui fait la matière de notre pratique théâtrale, c'est la matière elle-même ${ }^{2}$ ». Cette centralité de la matière fait des arts de la marionnette un terrain privilégié d'expérimentation et de réflexion - et possiblement de critique - autour des enjeux liés à l'animation, l'inertie et l'agentivité. Au-delà de la possible symbolique associée à chaque matériau, au-delà de sa sémiotique (que nous prenons toutefois en compte), ce sont aussi - et peut-être surtout - ses caractéristiques physiques singulières qui nous intéressent : poids, souplesse, élasticité, résistance à la chute, à l'arrachement, à la torsion, dureté, texture, teinte, odeur, potentiel de transformation d'un état à un autre et, de façon tout aussi tangible, mais moins scientifiquement quantifiable, sa vibration, l'empreinte qu'il laisse au creux de la paume. Cette physicalité propre à chaque matériau possède un potentiel théâtral, un champ lexical, des résonances expressives et signifiantes pour l'artiste et le public (possiblement différentes pour l'une et l'autre). 
3 Eric Bass donne un exemple concret de la façon dont - même dans le contexte d'une représentation anthropomorphe qui ne remet pas en cause la centralité de la figure humaine - la matière qui compose la marionnette permet de transposer les enjeux d'une pièce. "Si le dialogue parle de s'envoler, les marionnettes peuvent-elles être faites de pierre, suggérant ainsi la lourdeur, de façon à ce que nous nous projetions de façon accrue dans le besoin de s'envoler ? ${ }^{3}$ ». Dans une telle proposition, les limitations et les propriétés physiques de la pierre contribueraient à rendre intelligibles et sensibles les aspirations du personnage et pousseraient le public à y investir son attention et son empathie. C'est là faire appel à la connaissance somatisée progressivement inscrite dans le corps - qu'a chacune du poids et de la physicalité de ce matériau, à ses sensations passées, à son expérience de vie, à son histoire d'interactions diverses avec les roches et les cailloux, gros et petits, lancés, jetés ou laissés tomber. En d'autres termes, c'est créer une empathie kinesthésique qui fait appel au corps de la spectatrice et du spectateur comme porteur d'expériences physiques et psychiques singulières. C'est aussi en partie cet aspect mnésique qui explique notre attachement à la marionnette : elle fait appel à nos ressentis incorporés et sollicite des sensations souvent très quotidiennes et détachées de toute virtuosité, du moins en apparence. Elle nous ramène également au cheminement expérimental propre aux apprentissages de l'enfance, âge où l'on fait face au mystère des choses et de leur fonction(nement). Nous avons toutes eu à comprendre comment interagir avec le réel par le biais de notre propre corps, lui-même à découvrir. Nous avons été prêt-es à tous les échecs, tous les essais successifs pour, selon nos expériences et les capacités de nos corps, vaincre la gravité qui nous tenait assises, trouver la courbe de la cuillère pour qu'elle se rende dans notre bouche, ou comprendre dans la douleur la différence entre la moelleuse malléabilité des jouets qui font squish ${ }^{4}$ et l'angulaire brutalité du coin de table (ce ne sont que quelques exemples, tous les corps ne font pas ces expériences précises pour diverses raisons).

4 Eve Kosofsky Sedgwick écrit de façon limpide dans Touching Feeling ce que le sens du toucher induit :

Percevoir une texture c'est savoir ou conjecturer qu'une chose sera facile ou difficile, sans risque ou dangereuse à attraper, empiler, plier, déchirer, escalader, étirer, faire glisser, tremper. Plus encore que les autres systèmes perceptifs, il semble que le sens du toucher rende absurde toute compréhension dualiste de l'agentivité et de la passivité ; toucher c'est toujours déjà tendre vers, caresser, soupeser, taper, envelopper, et c'est également toujours comprendre que d'autres personnes ou forces naturelles ont bel et bien fait de même avant nous, ne serait-ce qu'au cours de la fabrication de l'objet texturé. ${ }^{5}$

5 Parce que nous, marionnettistes, créons et/ou choisissons nos objets et matériaux avec une attention toute particulière prêtée à ce que Kosofsky Sedgwick appelle leur "texture ", nous plaçons la sensorialité et notre relation à la matière au centre de notre pratique. Plus encore, nous entrons en négociation permanente avec elle, y compris avec son inertie. J'irais jusqu'à dire que l'inertie est pour nous une modalité du mouvement. Lorsque l'on anime une marionnette, quel que soit son degré d'anthropomorphisme, on utilise son poids, sa réaction à la gravité et l'ensemble de ses propriétés physiques. On cherche les points par lesquels lui transmettre l'impulsion avec efficacité, l'économie générale de son mouvement, la façon dont certains membres ou segments peuvent bouger par inertie (cela est vrai à tous les stades, de la fabrication à l'animation). Pour reprendre le beau titre du chorégraphe Ushio Amagatsu, donner 
vie à une marionnette est un «dialogue avec la gravité » et cela suppose une connaissance des matériaux et de la façon dont ils réagiront aux impulsions d'animation. La mousse ne répondra pas de la même façon que le bois, un membre de dix centimètres n'aura pas le même ballant qu'un autre de dix mètres, la force à exercer variera dans sa qualité. Depuis la conception jusqu'à la représentation, il est donc impossible de faire fi des questionnements liés à la matière dans une démarche de création marionnettique.

6 Qui plus est, ces interactions constantes avec la ou les matières qui constituent la figure marionnettique ont un effet en retour sur le corps de l'interprète - peu importe que l'on ait affaire à un matériau brut, un objet manufacturé ou une marionnette anthropomorphe. Ce contact des corps - corps de chair et corps constitués d'autres matières, corps dans leur physicalité tangible et possiblement changeante, corps réels et fictifs (mais tous les corps en scène ne sont-ils pas fictifs? Et qui peut dire qu'un corps en bois ou en silicone est moins réel qu'un corps humain?) - demeure in fine au cœur de toute démarche marionnettique.

7 Si la puissance de la marionnette tient évidemment d'abord à notre incessante projection, comme artistes et comme public, de nos propres affects sur tout ce qui nous entoure, à notre paréidolie qui voit des visages jusque dans les cailloux, doit-on penser que cette tendance ne parle que de notre hybris humain qui croit se voir partout en "maître et possesseur ${ }^{6}$ ? Ne nous parle-t-elle pas aussi de notre place dans un écosystème auquel nous appartenons et qui nous dépasse? Autrement dit, la mise en scène de l'humain face à - ou aux côtés de - la matière ne nous parle-t-elle pas fondamentalement, au-delà de notre capacité à la transformer et à en maîtriser la résistance, de notre propre matérialité, de notre corps parmi les corps, de notre impermanence (transience)?

\section{Premier interlude : mémoires de la matière}

8 J'ai décidé de prendre des cours de céramique. C'est une façon de renouveler mon contact avec un matériau qui m'accompagne depuis l'enfance, puisque mes parents étaient céramistes. J'ai grandi avec la terre, l'argile, dans tous ses états : sa poussière en suspension jusque dans la cuisine; les tas de terre molle à recycler attendant, près du poêle, de sécher suffisamment pour être pétris, homogénéisés, puis utilisés à nouveau; les pièces rougeoyantes à l'odeur de sciure de bois, que l'on extrait du four avec de longues pinces en métal ; la barbotine sur les doigts qui fait une peau de crocodile; les pièces ratées ou considérées telles, réduites en tessons tassés sur la parcelle du champ qui sert à garer la voiture, pour que ses roues ne s'embourbent pas les jours de pluie.

9 Je sors de mon cours de tournage, qui ramène tous ces souvenirs d'enfance à la surface, émue d'apprendre que l'argile aussi a une mémoire.

10 Mes pièces se sont déformées lors d'un séchage contraint, trop proches les unes des autres. Je leur ai, avec délicatesse et persévérance, redonné leur rotondité avant la première cuisson. Biscuitées, elles semblaient parfaites. Je les ai émaillées, persuadée d'avoir vaincu un matériau récalcitrant. Après la deuxième cuisson, la terre a repris et figé les déformations: mes bols croches étaient redevenus croches. Exposée à de fortes températures, elle s'est «souvenue» des tensions et torsions, et les a révélées à nouveau. La terre garde l'empreinte des formes qu'on lui donne. 
11 Moi qui recycle chaque fois mon argile une fois la sculpture moulée, modelant et remodelant têtes et mains, et parfois pieds, membres ou bustes, dans la même motte de terre, souvent pendant plusieurs années : est-ce que je travaille avec un matériau chargé de toutes ces formes? Conserve-t-il une trace infime, à un niveau moléculaire, de toutes ces incarnations, tous ces visages que je lui donne? Est-ce que cela change progressivement la façon dont il se plie à-ou au contraire guide - mes idées et mon imaginaire?

\section{2. «Material performance »}

12 J'aime ce terme de material performance apparu récemment dans les recherches anglophones afin de nommer tout un pan de la création contemporaine - plus ou moins ouvertement marionnettique - qui se propose de faire du potentiel expressif et signifiant de la matière non seulement un champ d'exploration, mais également une modalité de création.

13 Dassia N. Posner définit en ces termes le concept dans son introduction à la précieuse compilation d'articles The Routledge Companion to Puppetry and Material Performance :

« une performance qui suppose que la matière inanimée contient une agentivité permettant non seulement de mimer ou de refléter, mais aussi d'informer et de créer ${ }^{7}$.

14 On perçoit la proximité avec la marionnette - définie quant à elle comme « effet de vie autonome que les humains projettent, dans le cadre d'un spectacle, sur des objets dépourvus de vie, mais non d'agentivité ${ }^{\prime} "-$, mais également le changement de paradigme : il ne s'agit plus tant de développer savoir-faire et maitrise que de s'éloigner de la figure du démiurge et de radicaliser une posture d'attention, on pourrait dire de care $e^{9}$, autrement dit de se mettre au service et au diapason de la matière pour en saisir les propositions. Il ne s'agit évidemment pas là d'une rupture totale, bien des habiletés de l'interprète-marionnettiste relevant déjà de cette capacité attentionnelle, mais le rapport à la virtuosité et à l'intentionnalité de l'artiste en est sensiblement transformé.

Il devient ici incontournable de faire un détour théorique par le champ de réflexions regroupées sous différentes appellations telles que «Nouveau(x) Matérialisme(s»), «Object-Oriented Ontologies» ou "Nonhuman Turn $»^{10}$, champ qui a un impact grandissant sur la réception critique des œuvres, notamment scéniques, ainsi que sur la réflexion et la pratique des artistes - comme en témoignent plusieurs publications au cours des dix dernières années, notamment anglophones. Je ne prétendrai pas être une spécialiste de ces champs de recherche, mais, depuis mon arrivée inopinée dans le monde universitaire, j'ai lu suffisamment de textes qui en étaient nourris et rencontré plusieurs artistes dont la pratique avait été transformée par certaines lectures (au premier titre le désormais classique Vibrant Matter de Jane Bennett ${ }^{11}$ ) pour m'y intéresser.

16 Il me faut néanmoins avouer que ces textes ne se laissent pas toujours appréhender aisément - après ma troisième lecture de Karen Barad, je ne suis toujours pas sûre d'avoir tout à fait saisi ce qu'est le réalisme agentiel ${ }^{12}$ - et que j'éprouve une certaine perplexité à découvrir la démultiplication des turns paradigmatiques du monde universitaire. Si certains d'entre eux font sens même pour la novice que je suis (quoiqu'ils s'accompagnent souvent de prétentions à faire rupture avec les recherches précédentes, prétentions qui paraissent parfois relever d'un geste rhétorique quelque peu outrancier $\left.{ }^{13}\right)$, d'autres s'apparentent pour moi à des discussions absconses dont je 
perçois mal en quoi elles m'offrent des outils pour rendre intelligibles ma pratique et ce qu'elle induit.

Malgré ces difficultés, ces textes - et plus encore la façon dont certain'es artistes s'en emparent- me nourrissent. Il faut dire que, de par la nature liminale de la marionnette, je travaille à cet endroit charnière où la chose et le personnage ne cessent de coexister en une même figure, et que cela me rend très sensible à toute pensée qui travaille à déconstruire les dualismes hérités, notamment, de Descartes, et la scission absolue entre objet et sujet, corps et esprit. Bien des écrits féministes, au premier plan ceux de Donna Haraway ${ }^{14}$, démantèlent brillamment et depuis déjà plusieurs décennies tout un ensemble d'oppositions structurelles de la pensée occidentale : nature/culture, science/art, objectivité/subjectivité, humain/animal, animé/inanimé, etc. Les pensées queer et décoloniales travaillent également à l'éclatement de ces couples binaires et critiquent les assignations limitantes qui résultent de leur imposition, souvent par la force, notamment lors des différentes vagues de colonisation. Je dois admettre que je trouve ces lectures passionnantes et souvent plus porteuses que celles que j'ai pu faire relevant plus strictement des Nouveaux Matérialismes, parce que leurs implications politiques sont plus nettes.

Le rapport renouvelé à notre environnement physique proposé par les Nouveaux Matérialismes ou le Nonhuman Turn ne m'intéresse en effet que s'il est à la fois poétisé qu'il trouve une inscription dans des œuvres - et surtout politisé - qu'il amène à un positionnement clair et écocritique, et à une véritable éthique de travail avec les matériaux dans un monde irrémédiablement abîmé par la logique extractiviste. Il y a un vrai risque d'apolitisme dans ces théories, tout d'abord parce que - comme le soulignent aussi bien Ahmed que Sofer ${ }^{15}$ - elles font souvent fi du matérialisme historique qui, pourtant, offre des outils fort intéressants pour questionner notre rapport au monde et aux choses que nous (y) avons créées et produites. Ensuite, parce que conférer aux choses et à la matière une place et une agentivité dans un monde dont l'organisation politique, économique et sociale repose encore entièrement sur le refus d'accorder ces mêmes qualités à une majorité d'être doués de sentience ${ }^{16}$ peut être éminemment problématique. Les critiques de Schneider, Chen et Sofer sont à ce titre fort convaincantes ${ }^{17}$. Comme le rappelle Chen, «le fait d'être considéré comme animé [doué d'une anima] est politique et informé par ce ou ceux qui comptent comme humains et ce ou ceux qui ne sont pas considérés tels ${ }^{18}$ », et la catégorie de ce qui est considéré animé, vivant, sain est organisée selon des logiques raciales, sexistes, spécistes ${ }^{19}$ et validistes qui dévaluent certains corps. Schneider prolonge cette réflexion en nous mettant en garde contre le risque qu'il y a, dans ce contexte, à « étendre de manière romantique la catégorie de vivant/animée ${ }^{20} »$. Sofer questionne quant à lui : «Pour bien des personnes démunies, y compris les presque 46 millions d'individus actuellement réduits en esclavage à travers le monde, l'agentivité au sens d'une autodétermination élémentaire demeure une aspiration. Pourrait-il être prématuré de conférer une agentivité à l'inanimé autant qu'à l'animé, à ce qui est doué de sentience aussi bien qu'à ce qui ne l'est pas ? À quel moment l'agentivité post- ou non-humaine devient-elle un signifiant apolitique - un simple synonyme d'énergie ? ${ }^{21}$ "

19 «I am not interested in policing boundaries between nature and culture - quite the opposite, I am edified by the traffic. $»^{22}$ (Donna Haraway) 


\section{Deuxième interlude : vers l'infini et au-delà ?} j'ai émise, à savoir que le personnage marionnettique résulte d'une forme de co-création par l'interprète et le public. Est-ce que, me demande-t-elle, les marionnettes ne seraient pas au contraire plus vivantes lorsque personne ne les regarde? Je suis perplexe. Je vois bien qu'il y a là pour elle quelque chose de philosophiquement beaucoup plus vaste et profond que le scénario de Toy Story (qu'on ne se méprenne pas, j'aime beaucoup Toy Story, notamment ce passage du troisième film de la franchise, qui confronte les héros à leur destruction imminente avec une honnêteté désarmante pour un film jeunesse) et que je ne saisis sans doute pas de quoi elle parle. Pour ma défense, sa question est tellement déconnectée de ce qui fait ma pratique artistique que je ne sais trop quoi répondre.

Ce qui m'étonne, aussi, c'est qu'imaginer que les marionnettes sont plus vivantes lorsqu'elles sont laissées à elles-mêmes, sans être animées ni même regardées, participe selon moi d'une sorte de fétichisation anthropomorphique de l'objet (il aurait une vie propre qui semble toujours plus ou moins envisagée comme un double de la nôtre). Or cela me semble aller à l'encontre de la volonté de décentraliser l'humain que revendique justement cette interlocutrice. Qui plus est, loin de m'émerveiller, je trouve que cette idée affadit le temps du spectacle. Non que j'imagine celui-ci comme étant de l'ordre du sacré, ni même du rituel ${ }^{23}$, mais l'idée que la marionnette se pare pendant la représentation de qualités qui ne précèdent ni n'excèdent la durée de celle-ci me fascine et m'émeut. agentivité que celui qui se déploie dans les textes fondateurs des Nouveaux Matérialismes et de leurs exégètes:

"Qui sait: peut-être que, sortis du moment «agentivité », nous pourrions nous retrouver avec un vocabulaire nouveau et plus précis - voire un verbe ou deux pour décrire les façons dont les objets scéniques, en tant qu'objets, jouent toujours pour nous, aussi tentant soit-il de les imaginer dansant par et pour eux-mêmes ${ }^{24} »$.

\section{Ces objets et matériaux que l'on met sur/en scène}

Outre les questionnements déjà mentionnés relativement aux Nouveaux Matérialismes et à leur utilité pour analyser et approfondir ma pratique artistique marionnettique, je m'interroge plus généralement sur la pertinence de ces théories lorsqu'elles sont appliquées à la matière ou l'objet en scène. J'ai en effet souvent l'impression qu'il est fait de ces idées, au sein de la recherche en arts, un usage pour lequel elles n'étaient pas pensées, ce qui peut créer certaines tensions. Il me semble que c'est le cas en ce qui concerne les arts scéniques, car le choix humain qui a présidé au fait que matière et/ou objet se retrouvent sur le plateau, au sein d'une représentation, modifie et déplace les enjeux, tout particulièrement au regard de la question de l'authorship ${ }^{25}$.

Si je suis très sensible, par exemple, à la démarche de la chorégraphe Thea Patterson ${ }^{26}$, qui recherche une éthique relationnelle entre interprètes et matière, et tente de faire disparaître le geste humain de son écriture chorégraphique, je ne peux faire fi des nombreuses questions que son travail me pose. Il est sans doute normal qu'une artiste qui, comme moi, est intéressée par la compréhension du monde à travers le prisme humain ${ }^{27}$, éprouve une certaine résistance face à une telle proposition artistique qui tente au contraire d'échapper à l'emprise de la subjectivité humaine. Ce qui me touche 
dans son travail est donc sans doute très différent de l'effet qu'elle recherche. Par exemple, dans Silver/d'Argent (2018 - en création), je m'attache à suivre toutes les réactions en chaîne (y compris ratées ou interrompues), à regarder la façon dont, par exemple, une traction continue sur un fil argenté entraîne un ensemble de modifications dans l'agencement des objets en scène. J'aime l'étrange intentionnalité de ses boules noires asymétriques qui, parfois, roulent avec une sorte de maladresse comique, avancent en quelque sorte d'un pas lourdaud, et parfois, semblant mues d'une soudaine impulsion, accélèrent et se rendent, seules, par une courbe imprévisible, à un endroit arbitraire de l'espace. Autrement dit, je projette sur ces objets sinon un personnage, du moins une figure avec des caractéristiques de locomotion animale. Chassez la marionnettiste, elle revient au galop!

\section{Photographie 1}

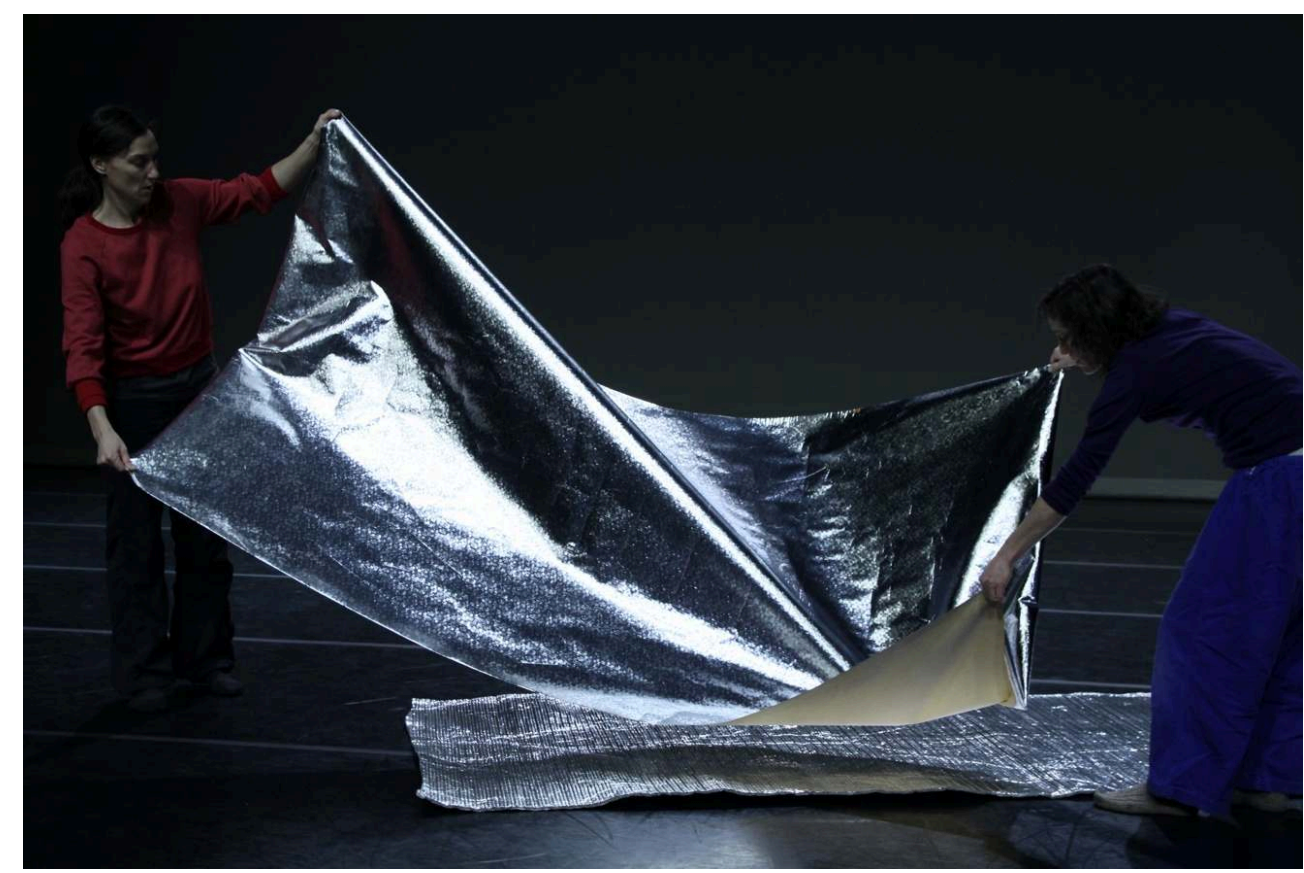

Silver/D'Argent (pièce en création). Chorégraphie de Thea Patterson. Interprètes : Rachel Harris et Elinor Feuter. Photo de Sigrid Patterson.

J'ai donc, face à ce type de proposition, deux interrogations majeures: tout d'abord, est-il possible de s'abstraire tout à fait du prisme de sa propre subjectivité humaine (en tant qu'artiste, d'abord, et a fortiori dans la réception du spectacle) ? Et, d'autre part, dès lors que l'on choisit les objets/matériaux qui seront en scène et qu'ils sont donnés à voir à un public, est-il vraiment possible de penser que l'authorship leur appartient de façon substantielle, quand bien même ce choix semble se faire au hasard ou parce que les objets semblent s'imposer à l'artiste? Pour reprendre l'exemple de la pièce de Patterson, les objets qui lui ont « fait de l'œil $\aleph^{28}$ sont argentés et/ou brillants (ce qui a déjà tendance à « attraper » le regard) et le fait de les choisir procédait d'une décision de se concentrer sur de telles qualités. Si le concept d'intra-action ${ }^{29}$ de Karen Barad auquel fait référence Patterson est fort séduisant pour traduire, notamment, tous les moments où objets et matériaux se font ingouvernables (unruly) et, échappant à notre maitrise, semblent posséder leur propre agentivité, il me semble tout de même qu'il se heurte rapidement à des limites dans ce contexte, dans la mesure où l'on ne peut 
affirmer que les différents corps en scène ne préexistaient pas à leur mise en relation, ni nier qu'ils ont fait l'objet d'un choix délibéré, de la part de l'artiste, de les placer en scène : il n'y ont pas surgi d'eux-mêmes et une certaine forme de hiérarchie s'inscrit déjà dans ce choix, qu'on le veuille ou non. Il a fallu discriminer certains objets au bénéfice de ceux qui sont finalement dans le « casting ».

Je tiens cependant à souligner que mes réticences à faire une application directe de ces théories aux arts scéniques ne s'apparentent pas à un rejet : je reste convaincue de leur pertinence pour sensibiliser aussi bien le public que les artistes à la matérialité de leurs dispositifs et pratiques, ainsi que pour élargir les modes d'entrée dans - et d'interaction avec - l'œuvre. Les textes de Rebecca Schneider et d'Amelia Jones ${ }^{30}$ font d'ailleurs désormais partie des incontournables de mes corpus, d'autant que leur approche est nourrie non seulement des Nouveaux Matérialismes, mais également des théories féministes et queer, et propose une synthèse brillante des enjeux dont j'ai parlé ici. Ces textes sont selon moi majeurs, car ils amènent à dépasser une posture d'adhésion ou de rejet face aux théories des Nouveaux Matérialismes et à complexifier notre rapport aux propositions artistiques. Ils nous engagent à conserver - en tant qu'artistes, mais aussi critiques - une forme d'humilité bienvenue face à la matière et aux processus mis en œuvre avec elle, à accepter de se dessaisir, au moins partiellement, de la maîtrise, y compris celle de la signification, toujours ouverte, voire flottante, au sein des œuvres, et à valoriser la place de la matière et du public dans l'écriture et la réception du spectacle dans un processus partagé de construction du sens. Il me semble en effet essentiel de ne jamais sous-estimer à quel point un objet artistique est, à plus d'un titre, relationnel, tout particulièrement au théâtre.

« Hovering between actor and prop, sign and substance, mimesis and kinesis, liveness and deadness, the staged object stands as a handy synecdoche for performance itself. » ${ }^{31}$ (Andrew Sofer)

\section{Troisième interlude : une histoire de peau, de terre et de métal}

Margaux Lecolier, une des étudiantes que j'encadre à la maîtrise en théâtre, travaille autour de deux figures féminines de Kleist traversées par le sentiment amoureux. Face aux interprètes, contre elles, deux matériaux très différents sont expérimentés pour évoquer le corps de l'homme aimé. L'argile rouge évoque celui qui, dans une rage amoureuse destructrice, a été déchiré (Penthésilée), sans que la terre soit jamais traitée de façon figurative. Dans l'autre exploration, un homme tout de grillage métallique, entièrement vide en-dedans, donne forme à un comte Von Strahl (La petite Catherine de Heilbronn) aussi fantomatique que tangible et qui devient prison dès que la lumière le traverse et projette l'ombre du matériau sur le visage de l'interprète. 
Photographie 2

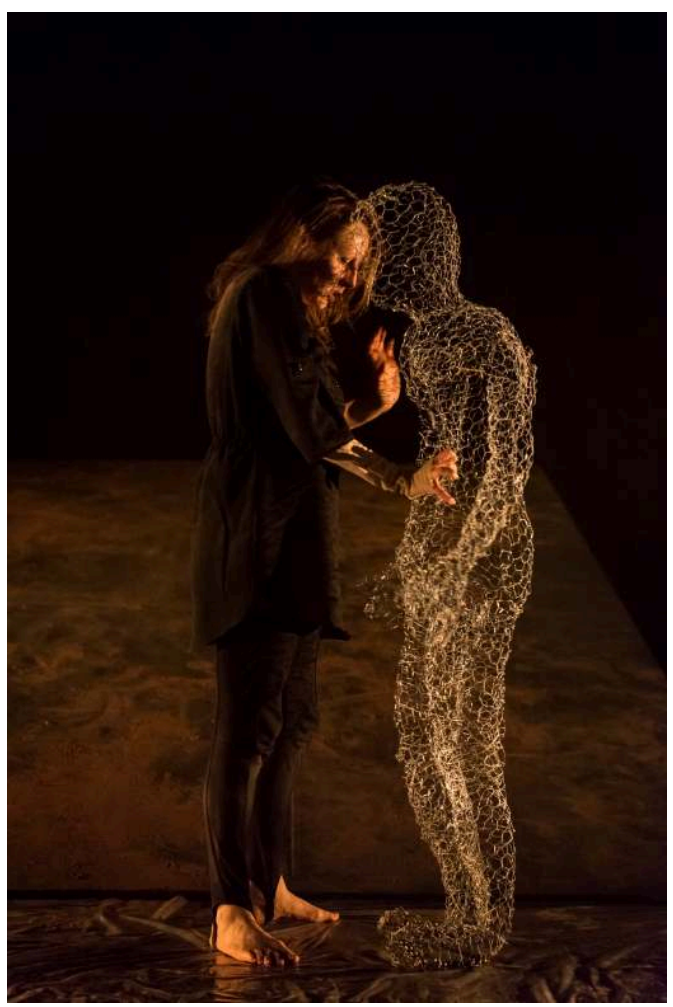

Cœur À Corps, conférence performative de Margaux Lecolier dans le cadre la maîtrise en théâtre. Université du Québec à Montréal, École supérieure de théâtre, mai2019. Interprète : Lucile Prosper. Photographie de Patrice Tremblay.

29 Ce qui m'intéresse, comme accompagnatrice du travail, n'est pas tant le résultat final que le processus de découverte par lequel passe l'artiste-étudiante : ce qui est juste ou non, ce qui est possible ou pas. Elle découvre notamment que le matériau brut (ou à peine mis en forme) permet de dire et faire à la fois (ce qui est souvent considéré comme un interdit au théâtre), de donner au public la " même " chose deux fois, une par les mots (ou les mouvements), une par les actions sur et avec la matière, car cette apparente redondance n'en est pas une dans ce type de travail. L'étudiante évoque aussi à plusieurs reprises à quel point le processus de création devient "organique" lorsque l'on décentre le texte. Tous les éléments trouvent alors leur nécessité et leur organisation, le texte devient matériau au même titre que le reste : les interprètes, le mouvement, la lumière, le son, la matière. Leur place et leurs interactions acquièrent peu à peu un statut d'évidence : l'ensemble fait corps.

Il y a quelque chose de beau et de touchant à voir une artiste en réflexion s'émouvoir de ce qui nous émeut, découvrir pour elle-même ce que l'on a aussi expérimenté, d'abord avec surprise, ensuite avec un émerveillement toujours renouvelé. Cela n'est pas affaire de satisfaction narcissique ou volonté de modeler les étudiant·es à son image. C'est être la témoin privilégiée de notre immédiate et troublante intimité - connue et pourtant chaque fois singulière-avec ce que Brian Jones (faisant lui-même un emprunt à l'architecte finnois Juhani Pallasmaa) appelle le texte haptique ${ }^{32}$ de la marionnette. 


\section{Matérialité d'un dispositif technologique}

31 Je reviens en France pour travailler avec la compagnie bordelaise La Boîte à Sel sur sa création BLOCK. Ce sont des retrouvailles : en mai 2016 avaient eu lieu les tout premiers laboratoires d'exploration à Montréal et à Québec. Depuis, les blocks sont passés de l'état de concept (un cube en Plexiglas ${ }^{\circledast}$ muni d'un petit haut-parleur qui pourrait émettre des sons) à l'état d'objets connectés réels, fabriqués par Thomas Sillard, munis chacun de sa propre carte son, d'un microprocesseur et d'un capteur de mouvement. Bien des laboratoires de recherche ont eu lieu sans moi, les blocks se sont multipliés jusqu'à atteindre la soixantaine, beaucoup d'idées et de scènes ont été trouvées, il s'agit maintenant d'écrire un spectacle, de concevoir une dramaturgie. En dix jours.

Il serait aisé de faire de ce travail une lecture à travers le prisme des Nouveaux Matérialismes, car ils ne sont guère dociles, ces blocks, à notre arrivée en salle. On peut même dire qu'ils sont particulièrement désobéissants. Chaque matin commence avec l'appel : il faut vérifier que chacun des soixante blocks répond présent. Bien sûr, il y a les mauvais élèves qui restent silencieux malgré les demandes réitérées, jamais les mêmes, jamais pour une raison identifiable. On pourrait célébrer « la puissance avec laquelle les choses et les objets écrivent, chorégraphient, dirigent, poussent, tirent, et de diverses manières animent leurs collaborateurs.trices humain $e^{33}$ » et font front contre l'hégémonie du sujet humain! Et à quel point celui-ci - en l'occurrence celle-ci, en la personne de Céline Garnavault, metteuse en scène et interprète du spectacle doit développer de nouvelles habiletés, « de nouvelles façons d'apprendre de, d'écouter et de collaborer avec des entités non-humaines ${ }^{34} »$. Autrement dit, il serait tentant, pour valoriser ce travail, d'en proposer une analyse philosophique qui ferait la part belle à l'agentivité du dispositif, mais ce serait occulter les efforts constants - et parfois angoissés - du créateur sonore pour que le système devienne aussi fiable que possible.

Cela reviendrait également à nier que la notion même d'écriture scénique, de dramaturgie, nous amène à structurer ce qui se passe sur scène d'une façon qui porte la marque de notre authorship. On peut le déplorer ou s'en réjouir, toujours est-il qu'en bout de ligne, il est attendu un spectacle qui, malgré des variations mineures, est censé rester essentiellement le même chaque fois ${ }^{35}$. Le travail est certes d'intégrer les accidents - que le dispositif technologique ne manque pas de produire et qu'il importe d'embrasser comme partenaire de jeu tout aussi faillible qu'une humainee, voire plus mais ces imprévus s'insèrent dans une courbe dramatique qui, partant de ce que les explorations ont révélé de possibilités d'interactions, les organise et propose une progression non seulement rythmique, émotive, mais également signifiante.

S'ajoute ainsi à la découverte des objets, du dispositif dans lequel ils s'inscrivent et des modes d'interaction et de création qu'ils permettent, la recherche de leur maitrise certes progressive et incomplète, mais toujours plus fine et précise. Sans entrer dans les détails techniques de conception des blocks et de leur fonctionnement, il a fallu de nombreux tâtonnements avant d'arriver aux objets tels qu'ils existent: les outils technologiques sont au service du spectacle et non l'inverse. Pourtant, le dispositif "dysfonctionne" régulièrement - du moins à ce stade de la création, on m'indique qu'il est, depuis cette résidence, devenu plus docile ! L'enjeu, lors de l'écriture, est donc de cesser d'envisager ces dysfonctionnements comme des ratés pour les accueillir comme des possibles, des propositions inattendues et riches. Des propositions néanmoins capricieuses, qui s'imposent à nous à des moments très différents du déroulé et, dans 
leur imprévisibilité, empêchent que nous les domestiquions tout à fait. Cela engage donc un type de présence singulier pour l'interprète, une ouverture à ce que les choses ratent, un rapport particulier à l'échec, évoquant le personnage burlesque qui peine à maîtriser un environnement récalcitrant.

Photographie 3

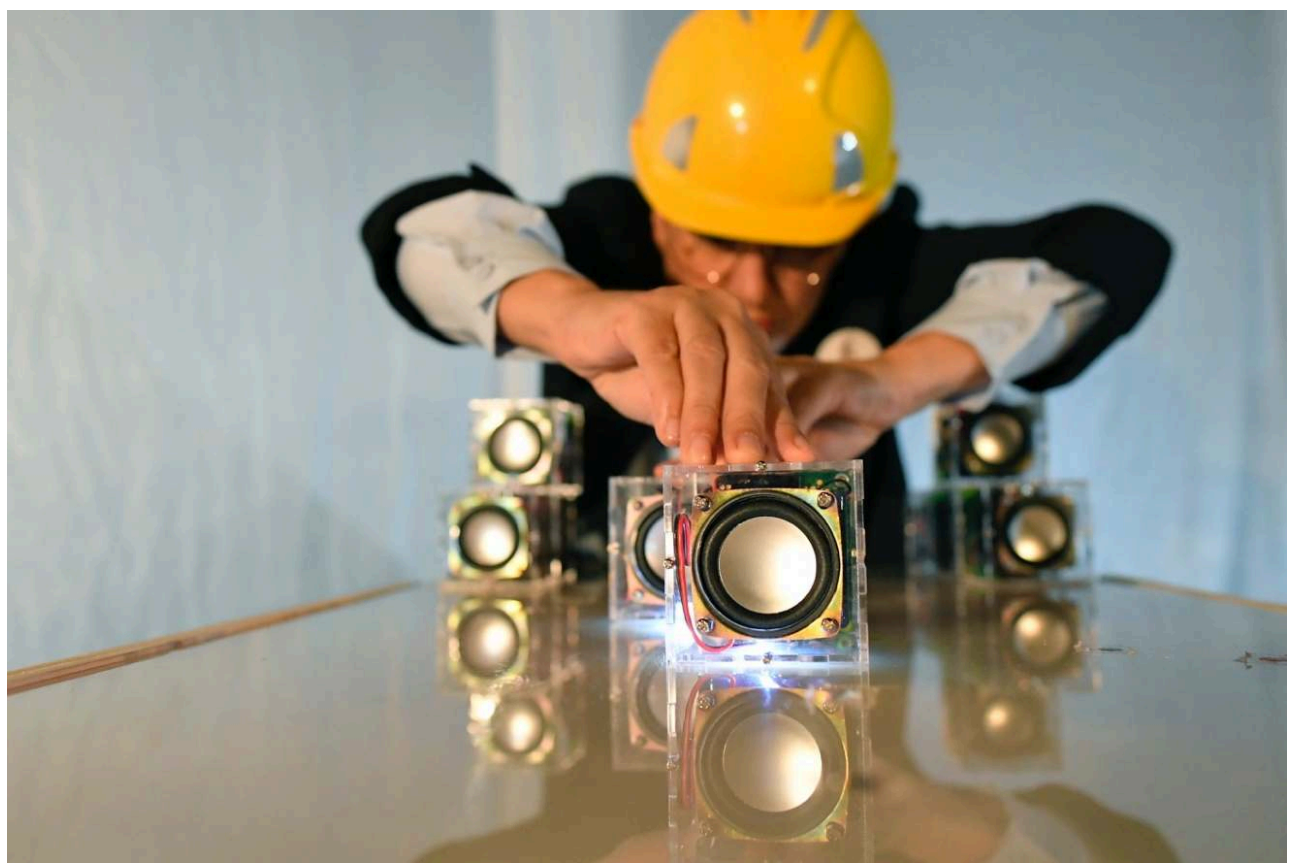

BLOCK, spectacle de Céline Garnavault et Thomas Sillard (compagnie La Boîte à sel, Bordeaux). Interprète : Céline Garnavault. Photographie de Frédéric Desmesures.

Du côté des artistes, c'est donc l'ouverture et la souplesse qui sont de mise, et un certain lâcher-prise. Du côté du public, invité à voir des morceaux du spectacle en construction, il y a un plaisir évident à assister au ratage, à la tentative de maîtrise et sa mise en échec par le dispositif, mise en échec parfois volontaire et écrite, parfois involontaire et provoquant quelques improvisations. Ce qui est néanmoins très intéressant, c'est qu'au-delà du plaisir, la réception semble très différente selon que l'on est face à des adultes ou des enfants (ceux-ci étant les premiers destinataires du spectacle). En effet, lors des présentations de chantier, une même angoisse est régulièrement exprimée par les adultes, malgré l'amusement : celle que la technologie nous échappe et nous engloutisse, humaines dépassées par les machines que nous avons créées. Les enfants, de façon fort intéressante, ne projettent pas cela sur la foule de blocks. Peut-être sont-ils beaucoup plus habitués à ce que le monde leur résiste, et disposés à continuer de jouer quoi qu'il en soit. Quant à moi, il me faut avouer que - à l'inverse de la personne qui prend en main la régie du spectacle ! - je trouve assez rassurant le fait que, aussi technologique et high tech soit-il, ce dispositif reste bien matériel, avec des bugs tout ce qu'il y a de plus analogiques (en plus des digitaux) liés notamment à la place de certains aimants, aux interactions inattendues avec les socles de métal qui forment des champs magnétiques, etc. Cela contribue à démystifier un peu le numérique comme technologie transparente et donne une couleur moins métaphysique à l'angoisse du dépassement : bien des choses nous échappent en effet, mais le résultat en est généralement plus comique qu'anxiogène et ne relève d'aucun 
projet machiavélique de machines toutes puissantes. De toute façon, comme nous le rappelle notre rituel quotidien de ramassage des blocks et leur branchement pour la nuit, rien de tout cela ne « vit » tant qu'on n'a pas rechargé les batteries.

\section{5. Érosions} Cette performance, souvent jouée in situ, est ici montrée en salle. Sur des socles faits de simples barres de métal de section carrée, l'artiste empile des pierres en de fragiles assemblages aux points d'équilibres ténus et improbables. Nous regardons l'œuvre en train de se faire, témoins et complices de la matière en transformation et de l'artiste à son écoute. L'œuvre est double : elle est à la fois dans les sculptures éphémères et dans leur lente réalisation à vue, dans cette expérience partagée qui cristallise le présent. Elle appartient au registre de ces œuvres hybrides dont parle Jones ${ }^{38}$, qui échappent à une lecture univoque soit en tant qu'objet d'art, soit en tant que performance. Les traces du processus de création sont elles-mêmes œuvres, aussi éphémères que soit leur durée (les pierres sont ensuite remises au sol, à la limite entre espace scénique et public, pour la représentation suivante, une fois que tout le monde a quitté la salle). L'œuvre interroge la relation entre l'action de l'artiste et la matérialité des pierres qu'il a choisies dans une carrière environnante. Tout comme l'œuvre de Cassils analysée par Jones, le travail de Steur

«illustre parfaitement un nouveau mode de pratiques hybrides qui puisent dans l'héritage du body art, de l'art conceptuel et de l'installation pour élaborer de nouvelles expériences artistiques complexes, qui sont performatives et existent pourtant sous diverses formes matérielles (incluant probablement le corps au travail de l'artiste) ${ }^{39}$."

Nick Steur joue avec nos nerfs, avec notre besoin de figer le résultat. Le spectacle tient en tension entre le faire et le fait. Il met à l'épreuve notre patience, et la sienne. En quoi est-ce un spectacle ? Est-ce un spectacle ? Il est fait spectacle, en tout cas, du risque, de la volonté de maîtrise et de sa faillite. 


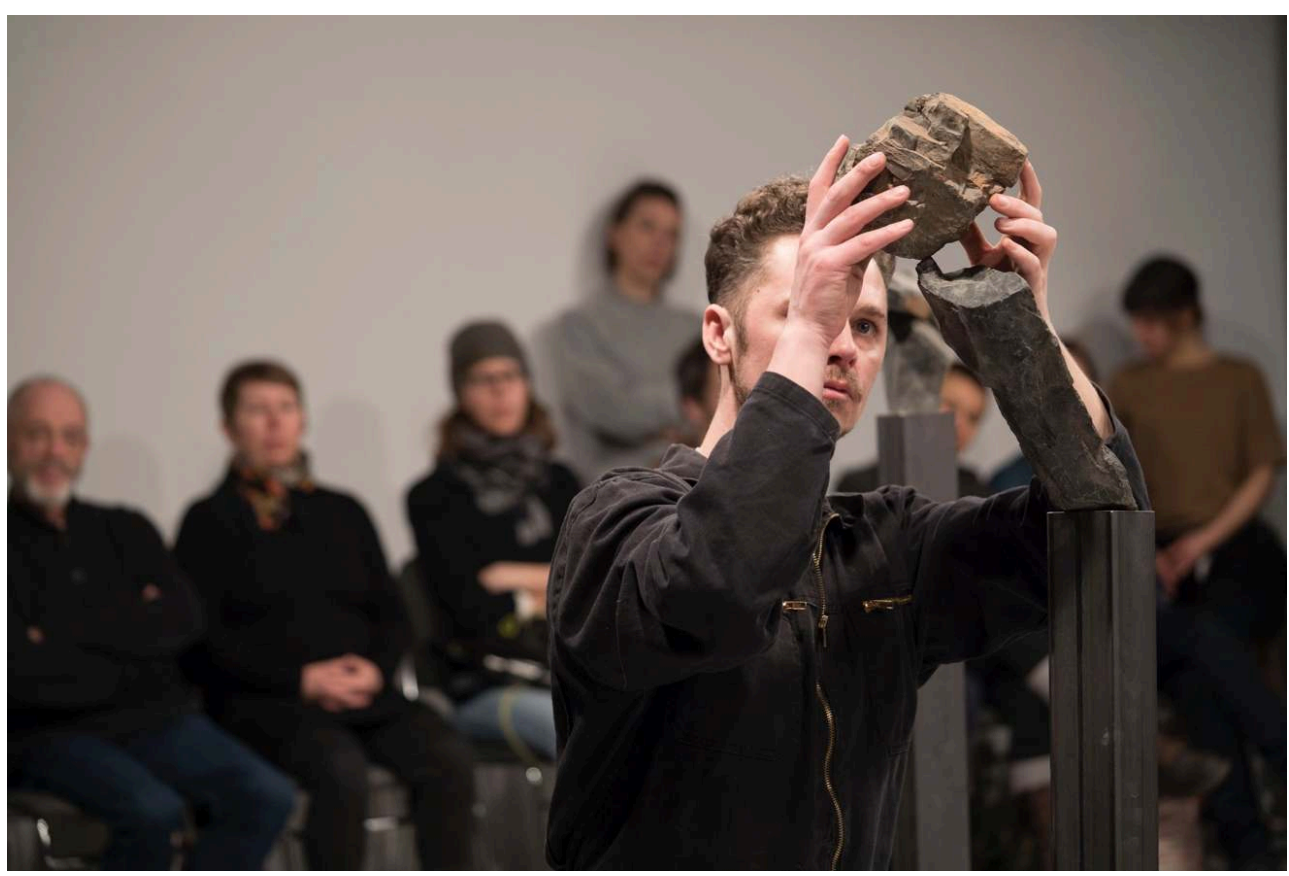

Freeze, performance de et avec Nick Steur. Festival Casteliers, Centre OBORO, mars 2018, Montréal Photographie de Josée Brouillard.

Il y a les échecs cuisants, dramatiques, qui coupent le souffle : lorsqu'un socle échappe des mains et que la vibration de sa chute à terre fait tomber quatre des six sculptures réalisées jusque-là. Mais le plus exigeant, ce sont les échecs lents, les échecs qui s'étirent, la friction interminable de la pierre sur la pierre, de cette infime surface de contact choisie justement parce qu'elle est si minime. C'est impossible, cela ne va pas tenir, prends donc plutôt cette autre surface, pourquoi chercher toujours l'équilibre le plus difficile? L'artiste prend une grande respiration et offre une de ses rares prises de parole: "Who here believes this is impossible? Come on, help me, I'm not doing this alone $^{40} »$. Quelques rires, francs ou embarrassés. C'est vrai que l'on commençait à s'impatienter, ça se sentait aux raclements de gorge discrets, aux subtils mouvements des corps qui tentent de répartir autrement leur poids sur la chaise. Steur prend le temps que cela prend. Il nous propose de prendre ce temps, de le perdre, de perdre jusqu'à sa notion. De ne pas regarder l'heure, de déposer nos inquiétudes de festivalières et festivaliers au planning serré de spectacles qui s'enchaînent. Le sien n'a pas de durée fixe, ni annoncée. Il faut l'accompagner. Et accepter de perdre. Accepter de s'éroder.

Il ne donne pas forme à l'informe. Il reconfigure temporairement des formes déjà existantes. Les résistances, les contraintes, les possibilités de la pierre sont source de satisfaction et de sens. Ces presque deux heures passées à regarder un artiste empiler des pierres sont tout à fait inutiles et absolument essentielles. Je repense à cet universitaire qui déclarait lors d'un colloque que, peut-être, le spectacle qui serait le plus subversif à l'heure actuelle serait celui qui prendrait le risque de proposer au public de s'ennuyer.

J'ai goûté la puissance d'être improductive. 


\section{En guise de conclusion temporaire}

41 Je n'en ai pas fini, loin de là, avec toutes ces réflexions. Chaque nouveau texte lu génère de nouveaux questionnements et me donne envie d'explorer plus avant, au plateau, avec la matière et les objets, cet étonnant assemblage humain-non humain que constitue la marionnette, au sens le plus large du terme. Ces textes me rappellent aussi pourquoi j'ai choisi ce médium, moi qui ai toujours eu la plus grande admiration pour les arts plastiques et visuels, mais n'ai jamais pu réaliser le moindre objet qui se serait suffi à lui-même. Créer un artefact, le mettre sur une étagère (ou autre réceptacle jugé adéquat) et dire "voilà, c'est mon œuvre", a toujours engendré chez moi des affres d'anxiété qui ne me permettaient pas d'arriver à un résultat pensé final. Sans doute suis-je encore trop sujette à la fétichisation des œuvres présente dans l'histoire de l'art - et ce malgré les critiques féministes qui lui ont été faites et auxquelles j'adhère. Déconstruire ses biais et apprentissages est une chose, cesser d'y souscrire dans l'intimité de son cheminement personnel en est hélas une autre. Les arts de la marionnette m'ont justement permis de trouver ma voie d'accès à une pratique visuelle et plastique, car l'objet n'y est pas pensé pour lui-même, mais pour la scène, pour ce moment où il sera amené à " vivre " pour un temps, sans que sa signification (et d'une certaine façon sa forme) soit arrêtée, fixe. La marionnette est créée pour le mouvement, pour l'espace de projection du plateau de théâtre qui impose une distance et un lieu. J'ai besoin de cela pour créer, c'est une certitude, même si je ne sais l'expliquer.

J'entame depuis quelques mois des études de Doctorat en Études et Pratiques des Arts à l'UQÀM, qui m'amèneront à créer, dans une perspective féministe queer, un essai scénique dans lequel l'argile aura une place centrale. Cette recherche-création fera également l'objet d'une thèse : autant dire que je n'ai pas fini de lire et de réfléchir à tous ces enjeux. Cela me met en joie, car je vois bien, à l'issue de cet assemblage fragmentaire et discontinu, que ma positionnalité d'artiste occidentale me pousse à mettre en doute, quoi que je fasse et aussi engagée que je sois à défaire les dualismes qui m'ont été inculqués, l'idée d'une réelle agentivité des objets et de la matière. Pourtant, je suis convaincue qu'il faut cesser de faire de l'humain et de sa subjectivité le centre de notre compréhension du monde et de nos pratiques, y compris artistiques, et que cette idée-même d'un centre ( $v s$ des périphéries) est limitée et limitante, voire dangereuse politiquement, notamment lorsqu'elle ouvre la voie à l'exploitation délétère de ce que nous appelons encore trop souvent "nos ressources». Il me faut donc m'engager maintenant dans des lectures à l'intersection entre Nouveaux Matérialismes, études environnementales et études autochtones. Il y a là, il me semble, un terrain fertile pour questionner plus encore cette culture coloniale à laquelle j'appartiens et que je prolonge (de façon involontaire, mais non moins réelle). L'argile de mes futures explorations vient d'un sol autochtone non-cédé et cela aussi fait partie de l'histoire sédimentée de ce matériau. 


\section{NOTES}

1. Voir l'article d'Eric Bass «Visual Dramaturgy, Some Thoughts for Puppet Theatre-Makers » in Dassia N. Posner, Claudia Orenstein and John Bell (eds.), The Routledge Companion to Puppetry and Material Performance, Routledge, Londres, Taylor \& Francis Group, 2015, pp. 54-60.

2. "As puppeteers, the material of our theatre-making is material itself", ibid., p. 55. Toutes les traductions depuis l'anglais sont celles de l'autrice.

3. Ibid., p. 57. Citation originale: "If the dialogue speaks of flight, can the puppets be made of stone, suggesting weight, so that our investment in the need for flight is enhanced?"

4. Onomatopée anglophone, correspondant aux petits bruits des jouets de bébé faits pour être écrasés.

5. Eve Kosofsky Sedgwick, Touching Feeling: Affect, Pedagogy, Performativity. Durham, Duke University Press, 2003, pp. 13-14. Citation originale: "[T]o perceive texture is to know or hypothesize whether a thing will be easy or hard, safe or dangerous to grasp, to stack, to fold, to shred, to climb on, to stretch, to slide, to soak. Even more immediately than other perceptual systems, it seems, the sense of touch makes nonsense out of any dualistic understanding of agency and passivity; to touch is always already to reach out, to fondle, to heft, to tap, or to enfold, and always also to understand other people or natural forces as having effectually done so before oneself, if only in the making of the textured object." (Je souligne).

6. René Descartes, «Sixième partie » in Discours de la méthode [1637], Paris : Garnier-Flammarion, 1966, p. 84.

7. «Introduction» in Dassia N. Posner, Claudia Orenstein et John Bell (eds.), The Routledge Companion to Puppetry and Material Performance, op. cit., p. 5. Citation originale : "[P]erformance that assumes that inanimate matter contains agency not simply to mimic or mirror, but also to shape and create."

8. Ibid. p. 5. Citation originale: "[H]uman infusion of independent life into lifeless, but not agentless, objects in performance."

9. Ce concept est développé à la fois dans certains courants de pensée féministe et dans l'étude des affects, les deux n'étant évidemment pas mutuellement exclusifs, comme le montrent les écrits de Sara Ahmed (voir notamment The Cultural Politics of Emotion, New York, Routledge, Taylor \& Francis Group, 2013). Le mot a en anglais une polysémie qui le rend difficile à traduire : à la fois soin, souci, affection, attachement, inquiétude, garde (responsabilité) et entretien.

10. Cette liste n'est pas exhaustive et n'implique nullement que ces différentes appellations reviennent au même. Au contraire, tout en partageant un engagement à décentrer l'humain aussi bien de la pensée que des pratiques de recherche, ces approches se trouvent en opposition quant aux formes que prend ce décentrement et quant à la façon d'envisager les objets et/ou la matière. Voir à ce sujet Richard Grusin (ed.), The Nonhuman Turn, Minneapolis, University of Minnesota Press, 2015.

11. Jane Bennett, Vibrant Matter, Durham, Duke University Press, 2009.

12. Voir l'article de Karen Barad "Poshumanist Performativity: Toward an Understanding of How Matter Comes to Matter» in Signs: Journal of Women in Culture and Society, vol. $28, \mathrm{n}^{\circ} 3$, University of Chicago, 2003, pp. 801-831.

13. Lire à ce propos les critiques adressées par Sara Ahmed aux Nouveaux Matérialismes quant à leur refus de s'inscrire dans un rapport de filiation avec nombre de travaux féministes majeurs relatifs à la biologie et au vivant, travaux dont l'occultation confine parfois à la malhonnêteté intellectuelle. Voir Sara Ahmed, "Some Preliminary Remarks on the Founding Gestures of the 'New Materialism' », European Journal of Women's Studies,. vol. 15, 2008, pp. 23-39. 
14. Voir, entre autres : Donna Haraway, Primate Visions: Gender, Race, and Nature in the World of Modern Science, (New York, Routledge,1989) et Staying with the Trouble: Making Kin in the Chthulucene (Durham, Duke University Press, 2016).

15. Voir Sara Ahmed, "Some Preliminary Remarks on the Founding Gestures of the "New Materialism' ", op. cit. ; et Andrew Sofer, "Getting on with Things: The Currency of Objects in Theatre and Performance Studies ", Theatre Journal, 2016, vol. 68, pp. 673-684.

16. Ce terme désigne le fait d'être en mesure de vivre des expériences et de les ressentir subjectivement: un être sentient ressent de nombreuses émotions, ainsi que la douleur et le plaisir. Ce qui lui arrive lui importe, et il tente d'éviter la souffrance et de mener une vie satisfaisante. Le terme est largement utilisé en éthique animale, car il permet de questionner les philosophies et pratiques qui font reposer les devoirs moraux envers autrui sur sa capacité de raisonner.

17. Voir Rebecca Schneider, "New Materialisms and Performance Studies ", TDR: The Drama Review, 2015, vol. 59, pp. 7-17. Andrew Sofer (art.. cit.) et Mel Y. Chen, Animacies. Biopolitics, Racial Mattering, and Queer Affect (Durham, Duke University Press, 2012) pour une analyse transversale relevant des études culturelles, décoloniales et queer.

18. Mel Y. Chen, Animacies..., op.cit., p. 30. Citation originale : "animacy is political, shaped by what or who counts as human and what or who does not."

19. Voir Yves Bonnardel, «Jouer les plantes contre les animaux », Libération, 17 avril 2019 (URL : <https://www.liberation.fr/debats/2019/04/17/jouer-les-plantes-contre-les-animaux_1721954>). Dans cette tribune, l'auteur déclare notamment: "Nous utilisons en permanence à tort des termes impliquant une subjectivité, un sujet conscient, pour décrire des processus. Ainsi parle-ton aujourd'hui de l'intelligence, de la mémoire, de la communication ou des stratégies des systèmes (informatiques, par exemple) ou des plantes, etc. Cela revient à décrire un thermostat comme « décidant » de chauffer la maison quand la température tombe en dessous d'un certain seuil. Bref, lorsqu'on vide de toute subjectivité des termes aussi fondamentaux que « conscience ", « intelligence », « communication», etc., ils ne désignent plus que des fonctionnalités. Ils en viennent à remplir le même rôle que la notion d'instinct autrefois : oblitérer la conscience des animaux, renvoyer leurs comportements à des mécanismes asubjectifs». Sur ces questions, voir aussi Yves Bonnardel "Chap. 4. Quelques réflexions concernant les plantes ", dans Yves Bonnardel, Thomas Lepeltier, Pierre Sigler (dir.), La Révolution antispéciste, PUF, 2018).

20. Schneider, op. cit., p. 13. Citation originale : "romantically expand the category of liveness".

21. Andrew Sofer, op. cit, p. 682. Citation originale : "For many disempowered persons, including the almost 46 million people currently enslaved around the world, agency in the sense of basic self-determination remains an aspiration. Might it be premature to confer agency upon the inanimate as well as the animate, the insentient as well as the sentient? At what point does postor nonhuman agency become an apolitical signifier - merely a synonym for energy?"

22. Donna Haraway, Primate Visions: Gender, Race, and Nature in the World of Modern Science, New York, Routledge, 1989, p. 377. Traduction: "Cela ne m'intéresse pas de réguler les frontières entre nature et culture - bien au contraire, ce sont les questions de circulation qui m'importent. »

23. Je ne me sens pas du tout légitime à user de ces termes qui ne correspondent pas à mon expérience ni à mes croyances, mais bien des personnes les emploient, car c'est ainsi qu'elles expérimentent le rapport à la marionnette, au masque ou à l'objet, ce qui me paraît alors évidemment justifié.

24. Voir Sofer, op. cit., p. 683. Citation originale: "Who knows : post-agency, we might find ourselves coming up with fresher and more precise language - even a verb or two - to describe the ways in which performing objects qua objects always perform for us, however tempting it may be to imagine them dancing by, and for, themselves". 
25. Je traduirais, de façon très insatisfaisante, par « autorité auctoriale ». Ladite insatisfaction est la raison pour laquelle je conserve le plus souvent le terme anglais dans ce texte.

26. Voir son site personnel : <https://theapatterson.wordpress.com>. Il est essentiel, pour leur faire justice, d'aller lire ses intentions artistiques qui sont bien plus articulées et intéressantes que le rapide résumé que j'en fais ici.

27. Voir Steve Tillis, Toward an Aesthetics of the Puppet, Westport, Greenwood Press, 1992, p. 56 : “ The audience is willing to imagine the puppet as having life because to do so fulfills the basic human desire to understand the world through the prism of human consciousness. The puppet's lack of consciousness as a perceived object is thus an invitation for the audience to participate in the creation of "life" similar to its own." (Traduction : « Le public est tout disposé à imaginer que la marionnette est vivante, car cela comble le désir humain fondamental de comprendre le monde à travers le prisme de la conscience humaine. L'absence de conscience propre à la marionnette en tant qu'objet perçu est de ce fait une invitation faite au public à participer à la création d'une "vie" similaire à la sienne. »)

28. La communication-démonstration de Thea Patterson à laquelle j'ai eu la chance d'assister avait pour titre «When Objects Wink at You» (symposium Moving Parts: Articulated Bodies and Objects in Performance, Université Concordia, 12 et 13 décembre 2018).

29. Le concept d'intra-action est un terme proposé par Karen Barad qui ne présuppose pas (contrairement au mot «interaction») l'existence de corps préexistants à l'action. C'est au travers de celle-ci que les corps se créent l'un l'autre dans un processus dynamique d'échanges et d'influences constants. Voir Karen Barad, art.cit.

30. Voir Rebecca Schneider, op.cit. et Amelia Jones, «Material Traces: Performativity, Artistic "Work", and New Concepts of Agency » in TDR: The Drama Review, vol. 59, n4, 2015, pp. 18-35.

31. "Oscillant entre interprète et accessoire, signe et substance, mimesis et kinesis, vie et mort, l'objet en scène se présente comme une métonymie commode de la représentation elle-même. » (Sofer, art. cit., p. 684).

32. Voir l'article de Basil Jones "Puppetry, Authorship, and the Ur-Narrative» in Dassia N. Posner, Ch Orenstein and John Bell (eds.), The Routledge Companion to Puppetry and Material Performance, op. cit., pp. 54-60. Jones s'intéresse à la question de l'authorship dans un spectacle de marionnette et donne l'exemple de la création de War Horse pour illustrer la façon dont le mouvement et les interactions avec la marionnette modifient de façon substantielle le script initial : "So ex post facto, the written text incorporates what, in fact, began as a movement text. This is what Juhani Pallasmaa might call "the haptic text" - the text of "active touch" and of the touching body moving through space." (Traduction: "Donc, rétroactivement, le texte écrit incorpore ce qui, en réalité, s'est initié comme écriture du mouvement. C'est ce que Juhani Pallasmaa pourrait appeler "le texte haptique" - le texte du "toucher actif" et du corps touchant qui se meut au travers de l'espace. »)

33. Voir Andrew Sofer, op. cit., p. 679. Citation originale: "the way that objects and things powerfully script, choreograph, direct, push, pull, and otherwise animate their human collaborators".

34. Ibid., p. 679. Citation originale : "new ways of learning from, listening to, and collaborating with nonhuman entities".

35. Il s'agit d'ailleurs d'une autre limite importante à la pertinence d'une grille de lecture inspirée des Nouveaux Matérialismes pour le spectacle vivant : contrairement à la performance dont il est entendu qu'elle est chaque fois unique et que ses éventuelles itérations comprendront de grandes différences, le spectacle tel qu'il est pensé - et acheté par les programmateur-trices est supposé rester globalement stable dans sa forme, son déroulement et sa durée.

36. Voir Rebecca Schneider, art. cit. p. 12. Traduction : «Le temps géologique est la temporalité dans laquelle vivent les pierres ». 
37. Le spectacle a été présenté à Montréal du 8 au 10 mars 2018 dans le cadre du festival Casteliers.

38. Voir Amelia Jones, art. cit., p. 18-35.

39. Ibid., p. 20. Citation originale: "epitomizes a new mode of hybrid practices that draw on a legacy of body, conceptual, and installation art to render new complex art experiences that are performative yet exist in various material forms (including, arguably, that of the artist's laboring body)."

40. Traduction: «Qui ici pense que c'est impossible? Allez, aidez-moi, je ne fais pas ça tout seul. »

\section{RÉSUMÉS}

Mêlant lectures, réflexions théoriques et expériences personnelles comme artiste, pédagogue et spectatrice, Dinaïg Stall interroge les modalités de son rapport à la matière dans le cadre d'une pratique marionnettique.

\section{INDEX}

Mots-clés : marionnette contemporaine, agentivité, nouveaux matérialismes, texture, authorship 